NASA Technical Memorandum 104343

\title{
Calculation of a Circular Jet in Crossflow With a Multiple-Time-Scale Turbulence Model
}

S.-W. Kim

University of Texas at Arlington

Arlington, Texas

and

T.J. Benson

Lewis Research Center

Cleveland, Ohio

July 1991

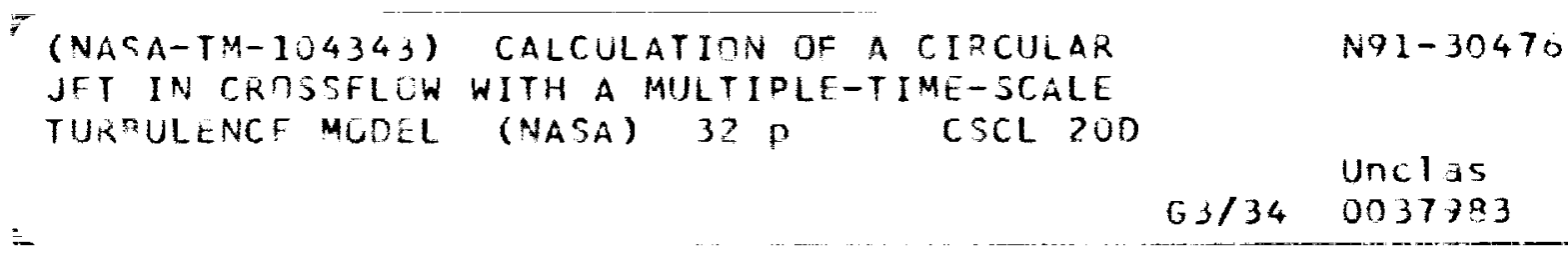




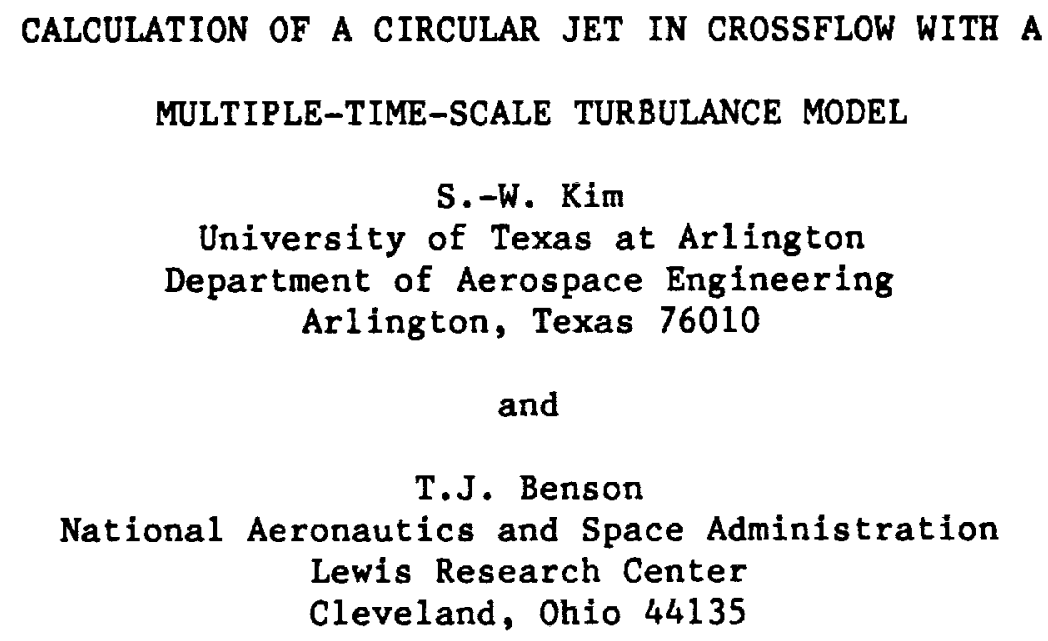

\section{SUMMARY}

Numerical calculation of a three-dimensional turbulent flow of a jet in a crossflow using a multiple-time-scale turbulence model is presented. The turbulence in the forward region of the jet is in a stronger inequilibrium state than that in the wake region of the jet, while the turbulence level in the wake region is higher than that in the front region. The calculated flow and the concentration fields are in very good agreement with the measured data, and it indicates that the turbulent transport of mass, concentration and momentum is strongly governed by the inequilibrium turbulence. The capability of the multiple-time-scale turbulence model to resolve the inequilibrium turbulence field is also discussed.

*NASA Resident Research Associate at Lewis Research Center. 


\section{$+\quad \therefore \quad-\ldots \quad$ NOMENCLATURE}

\begin{tabular}{|c|c|}
\hline$A_{j}$ & coefficient for $u_{j}$-velocity correction \\
\hline c & normalized concentration \\
\hline$c_{p} \ell$ & turbulence model constants for ${ }^{\epsilon} p$ equation $(\ell=1,3)$ \\
\hline$c_{t \ell}$ & turbulence model constants for $\epsilon_{t}$ equation $(\ell=1,3)$ \\
\hline$c_{\mu}$ & eddy viscosity coefficient \\
\hline$c_{\mu f}$ & constant coefficient $(-0.09)$ \\
\hline $\mathrm{D}$ & diameter of the circular jet \\
\hline $\mathbf{i}, \mathbf{j}$ & index for spatial coordinate $(i=1,2,3$ and $j=1,2,3)$ \\
\hline k & turbulent kinetic energy $\left(k-k_{p}+k_{t}\right)$ \\
\hline$k_{p}$ & turbulent kinetic energy in production range \\
\hline$k_{t}$ & turbulent kinetic energy in dissipation range \\
\hline $\mathrm{p}$ & pressure \\
\hline $\mathbf{p}^{\prime}$ & incremental pressure \\
\hline $\mathbf{P}_{\mathbf{r}}$ & production rate \\
\hline $\mathrm{U}_{\mathrm{o}}$ & free-stream velocity of crossflow \\
\hline $\mathrm{u}_{\mathrm{j}}$ & time averaged velocity $(-\{u, v, w\})$ \\
\hline $\mathbf{w}_{\mathbf{j}}$ & jet velocity averaged across jet cross-section \\
\hline$y^{+}$ & wall coordinate based on friction velocity \\
\hline${ }^{\epsilon} \mathrm{p}$ & energy transfer rate \\
\hline$\epsilon_{t}$ & dissipation rate \\
\hline$\mu$ & molecular viscosity \\
\hline$\mu_{\mathrm{d}}$ & molecular diffusivity \\
\hline$\mu_{t}$ & turbulent viscosity \\
\hline$\rho$ & density \\
\hline$\sigma_{\mathrm{d}}$ & turbulent Schmidt number \\
\hline$\sigma_{l}$ & turbulent Prandt 1 number for $\ell$-equation, $\ell=\left\{k_{p}, \epsilon_{p}, l\right.$ \\
\hline
\end{tabular}




\section{INTRODUCTION}

Numerical calculation of a circular jet exhausting into a cross flow [1] is presented. The circular jet in a uniform crossflow is schematically shown in Fig. 1. Turbulent flows similar to a jet in a crossflow can be found in a number of engineering applications. For example, in gas turbine combustors, a number of circumferentially distributed jets are used to ensure correct combustion in the flame zone and then to dilute the hot gas entering the turbine. Experimental investigations of air jets in crossflows have been made to better understand turbulent flows in such engineering applications even though air jets in crossflows are by far simpler than those in a gas turbine combustor. Compilations of various experimental investigations of jets in crossflows can be found in Crabb et al. [1] and Khan [2].

With the recent advances in numerical methods to solve the Navier-Stokes equations, a number of numerical simulations of jets in crossflows have been reported. A compilation of various numerical investigations of the flows can be found in Claus and Vanka [3]. Earlier numerical calculations of the flows $[4,5]$ have been devoted to the development and the verification of the numerical methods, and only a very small number of grid points were used to discretize the entire flow domain due to the limited capability of computers. More recently, a realistic number of grid points began to be used to calculate the flows using $k-\epsilon$ turbulence models [6]. The numerical results obtained using the relatively fine meshes show improved comparison with the measured data in a certain part of the flow domain and worse agreement with the measured data in the other part of the flow domain. Claus and Vanka [3] carried out a grid independence study of a jet in a crossflow using a $k-\epsilon$ turbulence model to identify the cause of 
the deteriorated comparison with the measured data. Claus and Vanka [3] showed that the numerical results obtained using a $128 \times 48 \times 48$ mesh are not significantly different from those obtained using a $256 \times 96 \times 96$ mesh and that the deteriorated comparison is caused by the Inability of the k- $\epsilon$ turbulence model to describe the complex turbulence field. In each of the above numerical simulations, the upstream region of the jet was excluded from the computational domain. However, Andreopoulos [7] showed that the circular jet and the crossflow interact strongly with each other at the jet exit and that the influence is propagated toward the upstream region of the jet. Thus, the deteriorated numerical results can also be caused by the numerical models which can not fully account for the strong interaction between the jet and the crossflow. In the present numerical study, the boundary for the circular jet is located at one diameter upstream of the jet exit so that the strong interaction at the jet exit is also accurately simulated.

Numerical results for various complex turbulent flows obtained using two-equation turbulence models, algebraic Reynolds stress turbulence models (ARSM) and Reynolds stress turbulence models (RSM) show that these turbulence models can not accurately describe the turbulence fields of various complex turbulent flows [8]. One common inability of the two-equation turbulence models, ARSM and RSM is that these turbulence models can not account for "inequilibrium turbulence" due to the use of a single time scale to describe both the turbulent transport and the dissipation of the turbulent kinetic energy. The "inequilibrium turbulence" is explained in the "Multiple-Time-scale Turbulence Model" section. The multiple-time-scale turbulence model [9] (hereafter abbreviated as the M-S turbulence model for conventence) ylelds accurate numerical results for 
widely different classes of complex turbulent flows (e.g., turbulent flows subjected to extra strains caused by streamline curvatures, interaction of multiple number of turbulence fields, and shock wave-turbulent boundary layer interactions). The complex turbulent flows to which the present M-S turbulence model has been applied as yet include a wall-jet flow, a shear layer with wake-boundary layer interaction, a backward-facing step flow, a confined coaxial swirling jet, turbulent shear layers over curved surfaces, separated transonic turbulent flows over a curved hill and reattaching shear layers in a divergent channel. It can be seen in [9-12] that the numerical results for these complex turbulent flows obtained using the $M-J$ turbulence model are in as good agreement with the measured data as those obtained using an optimized $k-\epsilon$, ARSM, or RSM turbulence model for each flow case. The capability of the M-S turbulence model to solve widely different complex turbulent flows is attributed to its capability to resolve the inequilibrium turbulence. This capability is discussed in the "Multiple-time-Scale Turbulence Model" section.

The fluid flow in the near-wall region of the jet exit is subjected to a large mean flow strain rate. The near-wall turbulence field, intensified by the large mean flow strain rate, can influence the entire fluid flow in the downstream region of the jet. Thus the near-wall turbulence field in the vicinity of the jet exit needs to be resolved accurately in order to correctly predict the entire flow field. In the present numerical simulation, the near-wall turbulence is described by a "partially low Reynolds number" near-wall turbulence model [13]. In the model, only the turbulent kinetic energy equations are extended to include the near-wall low turbulence region and the energy transfer rate and the dissipation rate inside the near-wall layer are obtained from algebraic equations. It was 
shown in numerical calculations of turbulent flows over curved hills [10], transonic turbulent flows over a curved surface with shock wave-boundary layer interactions [11], and reattaching shear layers in a divergent channel [12] that the "partially low-Reynolds number" near-wall turbulence model (when used together with the M-S turbulence model) accurately predicts the near-wall turbulence fields.

The present numerical method is a finite volume method based on a pressure correction algorithm [14-16]. In the method, all flow variables, except pressure and concentration, are located at the same grid points, while pressure and concentration are located at the centroid of a cell formed by the eight neighboring velocity grid points. The pressure correction algorithm is described in the following section. Calculations of a three-dimensional lid-driven cavity flow and a laminar flow through a $90^{\circ}$-bend square duct can be found in [15]. It is shown in [15] that the numerical results for the cavity flow obtained using the present numerical method compare more favorably with the measured data than those obtained using a formally third order accurate quadratic upwind interpolation scheme. It is also shown in [15] that the present method yields a grid independent solution for the curved duct flow with a very small number of grid points and that the method yields quickly and strongly convergent numerical results. Application of the same numerical method for two-dimensional flows can be found in [10-12].

NUMERICAL METHOD

The incompressible turbulent flow equations are given as;

$$
\frac{\partial}{\partial x_{j}}\left(\rho u_{j}\right)=0
$$




$$
\frac{\partial}{\partial x_{j}}\left(\rho u_{i} u_{j}\right)-\frac{\partial}{\partial x_{j}}\left\{\left(\mu+\mu_{t}\right)\left(\frac{\partial u_{i}}{\partial x_{j}}+\frac{\partial u_{j}}{\partial x_{i}}\right)\right\}=-\frac{\partial}{\partial x_{i}}\left(p+\frac{2}{3}-\rho k\right)
$$

where repeated indices imply summation over the index unless otherwise stated. The convection-diffusion equation for the concentration is given as ;

$$
\frac{\partial}{\partial x_{j}}\left(\rho u_{j} c\right)-\frac{\partial}{\partial x_{j}}\left\{\begin{array}{c}
\mu_{t} \\
\left(\mu_{d^{+}}+\frac{\partial c}{\partial x_{j}}\right)
\end{array}\right\}=0
$$

where $\sigma_{\mathrm{d}}=0.75$ is used in the present study. Due to the strong large eddy mixing, the molecular diffusivity can be ignored or formally approximated as $\mu_{\mathrm{d}}=\mu / \sigma_{\mathrm{d}}$; and neither of the approximations influence the numerical results significantly.

In the numerical method, the conservation of mass equation is replaced by a pressure correction equation given as:

$$
\frac{\partial}{\partial x_{j}}\left(\frac{1}{A_{j}} \frac{\partial p^{\prime}}{\partial x_{j}}\right)=-\frac{\partial \rho^{*} u_{j}^{*}}{\partial x_{j}}
$$

where $u_{j}$ * denotes the velocity which may not satisfy the conservation of mass as yet and the last term represents the mass imbalance (see [15] for details). The flow equations, the concentration equation, and the turbulence equations are solved by a finite volume method using the power-law upwind differencing scheme [14]. As all the central-differenced finite volume equations for self-adjoint second order elliptic partial differential equations are strongly diagonally dominant, the discrete pressure correction equation obtained by applying the standard finite volume method to eq. (4) is strongly diagonally dominant even for highly 
skewed and graded meshes.

For completeness, the velocity-pressure decoupling that occurs when various pressure correction algorithms are used for pressure-staggered meshes is described briefly below. The use of various pressure correction algorithms in pressure-staggered meshes does not yield a diagonally dominant system of equations for the incremental pressure. In such a case, the mass imbalance at a pressure grid point produces large corrections for pressures at adjacent pressure grid points and velocity-pressure decoupling occurs [15-16]. For clarity, the differences between the present pressure correction algorithm and various other algorithms are summarized below. For an orthogonal mesh aligned with cartesian coordinates, the present pressure correction algorithm and the "momentum interpolation scheme" of Peric et al. [17] and Majumdar [18] yield a 7-diagonal system of equations for the incremental pressure, while various other pressure correction algorithms yield a 27-diagonal system of equations which lacks diagonal dominance [15-16]. In the momentum interpolation scheme, the pressure gradient is interpolated differently from the other terms in the discrete momentum equation to achieve diagonal dominance. In curvilinear coordinates, the present pressure correction algorithm yields a 27-diagonal system of equations, while the momentum interpolation scheme always yields a 7-diagonal system of equations and it can not account for grid skewness in the discrete pressure correction equation in its present form. Also a specialized interpolation scheme needs to be adopted to obtain a unique solution that does not depend on the under-relaxation parameter [18]. On the other hand, the present pressure correction method yields a unique solution since the incremental pressure is driven only by the mass imbalance as shown in eq. (4). 


\section{MULTIPLE-TIME-SCALE TURBULENCE MODEL}

The anisotropy of the turbulence is the most easily detectable phenomenon in a measurement of a turbulent flow. Thus, it was conceived that the poor capability of the two-equation turbulence models to resolve complex turbulent flows is attributed to the inability of the turbulence models to take into account of the anisotropy of the turbulence. Thus the emphasis is laid upon improving the ARSM and the RSM. However, a number of numerical investigations carried out during the last one and half decades show that the ARSM and RSM still can not accurately predict the turbulence phenomena occurring in various flows unless the pressure-strain rate correlation is optimized for each flow [11-12].

A careful examination of semi-empirical data (theoretically derived data from a set of measured data) reveals that the inequilibrium turbulence also dictates the developments of the mean flow field and the turbulence field. Here, the "inequilibrium turbulence" represents the state of a turbulence field in which $\mathrm{Pr} / \epsilon_{\mathrm{t}}$ varies rapidly in space so that the shape and the frequency domain of the spectral density varies widely in space. The spectral density curves shown in Fig. 2-(a) are constructed based on the measured data of Klebanoff [19] and Wygnanski and Fiedler [20]. It can also be seen in Fig. 2-(a) that the generation of the energy containing large eddies by the instability of the mean fluid motion occurs in the low frequency region and that the peak of the spectral density moves toward the high frequency region as $\operatorname{Pr} / \epsilon_{t}$ is decreased. The semi-empirical $c_{\mu}$ for a plane jet obtained by Rodi [21] is shown in Fig. 3. It can be seen in the figure that $c_{\mu}$ is decreased as $\operatorname{Pr} / \epsilon_{t}$ is increased, and $c_{\mu}$ is increased as $\operatorname{Pr} / \epsilon_{t}$ is decreased. Thus, the developments of the mean fluid flow and the 
turbulence field are influenced by the spatially varying turbulent eddy viscosity, and the spatially varying turbulent eddy viscosity depends on the level of the local inequilibrium turbulence. Consider $k-\epsilon$ turbulence models for which the eddy viscosity is given as $\mu_{t}-\rho c_{\mu f} k^{2} / \epsilon_{t}$. Due to the use of a constant $c_{\mu f}$, the eddy viscosity in a strongly turbulent region $\left(\operatorname{Pr} / \epsilon_{t}>1\right)$ is over-predicted and that in weakly turbulent region $(\operatorname{Pr} \approx 0)$ is under-predicted. As an example, the under-predicted reattachment location of a backward-facing step flow obtained using a $k-\epsilon$ turbulence model is caused by the over-predicted turbulent viscosity along the reattaching shear layer [12].

The variation of $\mathrm{c}_{\mu}$ as a function of $\mathrm{Pr} / \epsilon_{t}$ was incorporated into $\mathrm{k}-\epsilon$ turbulence models in the form of "generalized algebraic stress turbulence madels." The $c_{\mu}$ curves by Launder [22] and Kim and Chen [23] are shown in Fig. 3. The generalized algebraic stress turbulence models yield accurate numerical results for shear layers when used in boundary layer flow solvers. However, the use of these turbulence models in elliptic (two-dimensional) flow solvers does not easily yleld a converged solution due to a severe interpolation used in the $c_{\mu}$ function. Furthermore, it is not clear if the generalized algebraic stress turbulence models can resolve the inequilibrium turbulence as cleanly as the M-S turbulence models can, since the generalized algebraic stress turbulence models lack many features of the M-S turbulence models to be described below.

The M-S turbulence models $[9,24]$ appeared as a consequence of recognizing the inability of various single-time-scale turbulence models ( $k-\epsilon$, ARSM, and RSM) to accurately describe complex turbulent flows. The convection-diffusion equations of the M-S turbulence models were established by Hanjelic et al. [24]. The convection-diffusion equations 
most naturally describe the physically observed turbulence phenomena in the sense that the turbulent transport of mass and momentum is described using the time-scale of large eddies and the dissipation rate is described using the time-scale of fine-scale eddies. Later, Kim and Chen [9] established the general form of the load functions based on a physical dimensional analysis. The differences between the present M-S turbulence model and that of Hanjelic et a1. [24] can be found in [11-12], and hence, these are not repeated here. The capability of the M-S turbulence model to solve complex turbulent flows was further enhanced by incorporating a "partially low-Reynolds number" near-wall turbulence model into the M-S turbulence model [10-13]. Calculations of widely different classes of complex turbulent flows showed that the M-S turbulence model can resolve the inequilibrium turbulence and can model the cascade of turbulent kinetic energy. These capabilities of the M-S turbulence model are described below.

The M-S turbulence equations are given below for completeness. The turbulent kinetic energy and the energy transfer rate equations for energy containing large eddies are given as;

$$
\begin{aligned}
& \rho u_{j} \frac{\partial k_{p}}{\partial x_{j}}-\frac{\partial}{\partial x_{j}}\left\{\left(\mu+\frac{\mu_{t}}{\sigma_{k p}}\right) \frac{\partial k_{p}}{\partial x_{j}}\right\}-\rho \operatorname{Pr}-\rho \epsilon p \\
& \rho u_{j} \frac{\partial \epsilon_{p}}{\partial x_{j}}-\frac{\partial}{\partial x_{j}}\left\{\left(\mu+\frac{\mu_{t}}{\sigma_{\epsilon}}\right) \frac{\partial \epsilon}{\partial x_{j}}\right\}-\rho c_{p} 1 \frac{\operatorname{Pr}^{2}}{k_{p}}+\rho c_{p} 2 \frac{\operatorname{Pr} \epsilon_{p}}{k_{p}}-\rho c_{p} 3 \frac{\epsilon_{p}}{k_{p}}
\end{aligned}
$$

where the production rate is given as;

$$
P_{r}=\frac{\mu_{t}}{\rho}\left\{2\left(\frac{\partial u}{\partial x}\right)^{2}+2\left(\frac{\partial v}{\partial y}\right)^{2}+2\left(\frac{\partial w}{\partial z}\right)^{2}+\left(\frac{\partial u}{\partial y}+\frac{\partial v}{\partial x}\right)^{2}+\left(\frac{\partial u}{\partial z}+\frac{\partial w}{\partial x}\right)^{2}+\left(\frac{\partial v}{\partial z}+\frac{\partial w}{\partial y}\right)^{2}\right\}
$$

The turbulent kinetic energy and the dissipation rate equations for fine 
scale eddies are given as:

$$
\begin{aligned}
& \rho u_{j} \frac{\partial k_{t}}{\partial x_{j}}-\frac{\partial}{\partial x_{j}}\left\{\left(\mu+\frac{\mu_{t}}{\sigma_{k t}}\right) \frac{\partial k_{t}}{\partial x_{j}}\right\}=\rho \epsilon_{p}-\rho \epsilon_{t} \\
& \rho u_{j} \frac{\partial \epsilon_{t}}{\partial x_{j}}-\frac{\partial}{\partial x_{j}}\left\{\left(\mu+\frac{\mu_{t}}{\sigma_{\epsilon t}}\right) \frac{\partial \epsilon_{t}}{\partial x_{j}}\right\}=\rho c_{t 1} \frac{\epsilon^{2}}{k_{t}}+\rho c_{t 2} \frac{\epsilon^{2} \epsilon_{t}}{k_{t}}-\rho c_{t 3} \frac{\epsilon_{t}}{k_{t}}
\end{aligned}
$$

and the eddy viscosity is given as;

$$
\mu_{t}=\rho c_{\mu \mathrm{f}} \frac{\mathrm{k}^{2}}{\epsilon_{\mathrm{p}}}
$$

The turbulence model constants are given as; $\sigma_{k p}-0.75, \sigma_{k t}=0.75, \sigma_{\epsilon}-1.15$, $\sigma_{\epsilon t}=1.15, c_{p 1}=0.21, c_{p 2}=1.24, c_{p} 3^{-1.84}, c_{t 1}=0.29, c_{t 2}=1.28$, and $c_{t 3^{-1.66}}$. The capability of the M-S turbulence model to resolve the inequilibrium turbulence depends largely on the load functions of the $\epsilon_{p}$ and $\epsilon_{t}$ equations and the way the turbulence model constants are established. The load functions of the $\epsilon_{p}$ and $\epsilon_{t}$ equations are obtained from a physical dimensional analysis [9], and the establishment of the model constants are based on the assumptions that the turbulence field of a uniformly sheared flow can approach an asymptotic state in which $\mathrm{Pr} / \epsilon_{\mathrm{t}}$ becomes a constant and that the ratio of $\epsilon_{t} / \epsilon_{\mathrm{p}}$ depends on the ratio of $\mathrm{Pr} / \epsilon_{t}$. The first assumption that such an asymptotic state can exist was shown by Harris et al. [25], and later, was confirmed by Tavoularis and Karnik [26]. In such asymptotic states of uniformly sheared flows, the diffusion term vanishes, and the asymptotic ratio of $k_{p} / k_{t}$ can be obtained by dividing eq. (5) by eq. (7), i.e.,

$$
\frac{k_{p}}{k_{t}}-\frac{D k_{p}}{D k_{t}}=\frac{\operatorname{Pr} / \epsilon_{t}-\epsilon_{p} / \epsilon_{t}}{\epsilon_{p} / \epsilon_{t}-1}
$$


It can be seen in eq. (10) that the existence of the asymptotic ratio of $k_{p} / k_{t}$ depends on the realizability of the second assumption. The second assumption that the ratio of $\epsilon_{t} / \epsilon_{p}$ depends on the ratio of $\operatorname{Pr} / \epsilon_{t}$ can be verified by numerical results posterlly, or it can be verified indirectly by comparing the M-S eddy viscosity equation with that of the generalized algebraic stress turbulence models shown in Fig. 3. The eddy viscosity, eq. (9), can be rewritten in a form compatible with that of the generalized algebraic stress turbulence mode1s, 1.e.,

$$
\mu_{t}=\rho c_{\mu} \frac{k^{2}}{\epsilon_{t}}
$$

where $c_{\mu}-c_{\mu f}\left(\epsilon_{t} / \epsilon_{p}\right)$ is the eddy viscosity coefficient, the variation of $c_{\mu}$ is described by the ratio of $\epsilon_{t} / \epsilon_{p}$, and $\epsilon_{t} / \epsilon_{p}$ is a function of $P_{r} / \epsilon_{t}$. Thus the second assumption can be Justified within the context of the generalized algebraic stress turbulence models.

The three inequilibrium turbulence levels ( $A, B$, and $C$ ) imbedded into the M-S turbulence model (or used in determining the turbulence model constants) are also shown in Fig. 3. The measured data that corresponds to the point A in Fig. 3 (i.e., $\mathrm{Pr} / \epsilon_{\mathrm{t}^{-1.5}}$ ) can be found in Harris et al. [25] and in Tavoularis and Karnik [26]. The value of $\epsilon_{t} / \epsilon_{\mathrm{p}}-0.95$ can be estimated from Fig. 3. The ratio of $k_{p} / k_{t}$ for the data point $A$ is obtained to be 9.0 from eq. (10). For turbulent flows in an equilibrium state (point B in Fig. 3), $\operatorname{Pr}=\epsilon_{t}$, and $\epsilon_{p}$ has to be equal to both of them to maintain the equilibrium state. In this case, eq. (10) becomes indeterminate; and the ratio of $k_{p} / k_{t} \approx 4.0$ can be obtained from a near-wall analysis of turbulent flows in equilibrium state [9]. In the free stream region of turbulent 
flows, the production rate vanishes. Such a case is represented by Point C in Fig. 3 and the ratio of $\epsilon_{t} / \epsilon_{\mathrm{p}} \approx 2.5$ can be estimated from the generalized algebraic stress turbulence model of Kim and Chen [23]. The ratio of $k_{p} / k_{t} \approx 0.7$ can be obtained from eq (10).

The three ratios of $k_{p} / k_{t}$ obtained in the above analysis show that

$$
\left(\frac{k_{p}}{k_{t}}\right)_{A}>\left(\frac{k_{p}}{k_{t}}\right)_{B}>\left(\frac{k_{p}}{k_{t}}\right)_{C}
$$

where $A, B$ and $C$ denote each turbulence state marked in Fig. 3 . The implication of the above inequality is illustrated in Fig. 2-(b), where the frequency domain is divided into two parts by the simplified split-spectrum $[9,24]$. The spectral density $A$ in Fig. 2-(b) belongs to a production dominated region $\left(P_{r} / \epsilon_{t}>1\right), B$ belongs to a near equilibrium region $\left(\mathrm{P}_{\mathrm{r}} / \epsilon_{\mathrm{t}} \approx 1\right)$, and $\mathrm{C}$ belongs to a region where production rate vanishes (i.e., free stream region). The spectral density curves $A, B$, and $C$ also describe the cascade process of turbulent kinetic energy that the energy containing large eddies (characterized by low frequencies) are generated by the instability of the mean fluid flow, the large eddies become finer eddies (characterized by higher frequencles), and the fine scale eddies are dissipated by the viscous force. It can be seen in Fig. 2-(b) that $k_{p} / k_{t}$ is greater for larger eddies and $k_{p} / k_{t}$ becomes smaller for finer-scale eddies. Thus the ratio of $k_{p} / k_{t}$ is determined by the shape and the frequency domain of each spectral density.

Calculations of various shear layers (boundary layer flows) using the M-S turbulence model always reproduce the imbedded inequilibrium turbulence states. For highly complex turbulent flows, large eddies generated in the upstream region are convected in the downstream direction: In this 
downstream region, the relationship between $\mathrm{P}_{r} / \epsilon_{t}$ and $\epsilon_{t} / \epsilon_{p}$ is influenced by the convected eddies (i.e., the large value of $k_{p} / k_{t}$ ) and the numerical results exhibit the trend of imbedded inequilibrium turbulence.

\section{NUMERICAL RESULTS}

The flow domain of the jet in a uniform crossflow [1] is shown in Fig. 1 . The jet velocity averaged across the cross-sectional area of the pipe is $27.6 \mathrm{~m} / \mathrm{sec}$, the free stream velocity of the cross flow is $12 \mathrm{~m} / \mathrm{sec}$, and the diameter of the jet is $0.0254 \mathrm{~m}$. In the experiment [1], the concentration field was measured by injecting helium gas (He) into the circular jet. The concentration of the helium is one percent of the air-helium mixture at the jet exit, and hence the concentration equation, eq. (3), is solved uncoupled from the momentum equations. The symmetric half of the flow domain is discretized by $148 \times 61 \times 94$ grid points in $x-, y-$, and $z$-coordinate directions, respectively. The body-fitted grid near the jet exit is shown in Fig. 4. The smallest mesh size in the direction normal to the wall is $0.6 \times 10^{-4} \mathrm{~m}\left(\mathrm{y}^{+} \approx 1.5\right.$ based on the fully developed pipe flow) and this mesh size is sufficiently small to resolve the near-wall turbulence field in the vicinity of the jet exit. The largest mesh size used near the far field boundaries is approximately half of the jet diameter.

The inlet boundary conditions for the tangential velocity, the turbulent kinetic energies, and the dissipation rates ( $\epsilon_{p}$ and $\epsilon_{t}$ ) are obtained from measured data for a fully developed boundary layer flow over a flat plate [19]. The non-dimensional velocity and the turbulent kinetic energy profiles are scaled to yield a boundary layer thickness of $0.005 \mathrm{~m}$ at the inlet boundary. The no-slip boundary condition for velocities, vanishing gradient for concentration, and vanishing turbulent kinetic energy are 
prescribed at the solid wall boundary. A vanishing gradient boundary condition is used for all flow variables at the exit boundary. The symmetric boundary condition is used on the south $(y-0)$ boundary, and the free stream boundary condition is used on the north $(y-3.1 D)$ and the top $(z=7.5 D)$ boundaries.

The bottom boundary is located at one jet diameter upstream of the jet exit (z--1D), and a fully developed pipe flow and a constant concentration $(c-1.0)$ conditions are prescribed as the fet inlet boundary. These boundary conditions can greatly reduce the uncertainty that can be caused by ill-posed boundary conditions at the fet inlet as discussed in the following paragraph. The use of the free stream boundary conditions on the north and the top boundaries is not a good approximation of the actual fluid flow unless these boundaries are located sufficiently far away from the jet exit. However, it can be seen in the following that the numerical results near the jet exit are not influenced too much by the far field boundary conditions [10]. The partition between the near-wall layer and the external region is located at $y^{+} \approx 100$ (based on the fully developed flat plate flow) and $10 \mathrm{grid}$ points are allocated inside the near-wall layer. The converged solutions are obtained in approximately 1200 iterations, and the relative mass and concentration imbalances are $2.5 \times 10^{-5}$ and $9.5 \times 10^{-3}$, respectively.

The contour plots of the jet velocity, the pressure, and the total pressure at the jet exit are shown in Fig. 5, where the increments between the contour lines are the same for each contour plot. It can be seen in the figure that the jet velocity, the static pressure, and the total pressure vary widely across the cross-section. It does not seem possible to prescribe a correct boundary condition for the jet if the bottom boundary 
is located at the jet exit. For example, in previous numerical calculations of jets in crossflows, either a constant vertical velocity or a constant total pressure was prescribed at the jet exit $[2,4]$. However, the present numerical results show that a significant amount of uncertainty can be caused by the use of either of these boundary conditions at the fet exit.

The calculated velocity vectors, pressure, turbulent kinetic energy; $\mathrm{Pr} / \epsilon_{t}, \epsilon_{t / \epsilon \mathrm{P}}$ and $\mathrm{k}_{\mathrm{p}} / \mathrm{k}_{t}$ are shown in Fig. 6. The velocity vector and the pressure contour plot show that the crossflow is decelerated rapidly by the jet and thus the pressure is increased in the forward region of the jet. Otherwise, the velocity vector and the pressure contour plot do not show that any significant phenomena occur in the forward region. However, the complex $\operatorname{Pr} / \epsilon_{t}$ and $\epsilon_{t} / \epsilon_{p}$ contours show that the turbulence field is experiencing an enormous evolution in the forward region. These contour plots show that the inequilibrium turbulence becomes stronger as a fluid particle approaches the jet and that the peak inequilibrium state occurs along the interface of the jet and the crossflow. The turbulent kinetic energy, the production rate, the energy transfer rate and the dissipation rate in the wake region of the jet are by far greater than those in the forward region of the jet. However, the turbulence in the forward region is in a stronger inequilibrium state than that in the wake region. These results indicate that the strength of inequilibrium turbulence does not necessarily depend on the turbulence intensity. It can be seen from eq. (11) that the rapidly varying $\epsilon_{t} / \epsilon_{p}$ in the forward region of the fet will influence the fluid flow significantly. It takes a while for large eddies to cascade to smaller eddies. The large ratio of $k_{p} / k_{t}$ in the wake region of the jet is caused by the large eddies convected from the upstream region and those generated in the wake region, see Fig. 6-(a). 
The calculated vertical velocity profiles in the vicinity of the jet exit are compared with the measured data in Fig. 7. The slightly higher w-velocity for $x / D>2$ is caused by the north boundary which is not located far enough from the jet exit. Due to the prescribed velocity profile (which is the same as that of the inlet plane) on the north boundary, the jet can not expand freely into the north direction and thus the excess mass flow rate along the symetry plane cause the over-predicted w-velocity in the region.

It is shown in Fig. 8 that the calculated tangential velocity profiles along the $x$-coordinate direction on the symmetry plane are in good agreement with the measured data. The reversed flow behind the jet indicate that the development of the tangential velocity along the crossflow direction is similar to that of the flow over a circular cylinder. However, the $u$-velocity in front of the jet is not brought to zero due to the compliance of the jet.

The u-velocity profiles at four downstream locations are shown in Fig. 9. The complexity of the u-velocity profiles are caused by the deflected jet and the separated crossflow behind the jet. It can be seen in the figure that the calculated results are in good agreement with the measured data qualitatively and quantitatively.

The calculated turbulent kinetic energy distribution along the $\mathrm{x}$-axis of the symmetry plane at $z / D-0.75$ is compared with the measured data in Fig. 10. It can be seen in the figure that the trend of the turbulent kinetic energy distribution is in excellent agreement with the measured data even though the turbulence intensity is under-predicted.

The calculated concentration profiles at three downstream locations on the symmetry plane are shown in Fig. 11, and the concentration contour plot 
at $x / d=8$ is shown in Fig. 12-(b). The shape and the peak locations of the calculated concentration profiles are in very good agreement with the measured data. The slightly smaller magnitude of the concentration is caused by the coarse grid inaccuracy in the far downstream region. For example, the concentration level near $(x / D, y / D, z / D) \approx(8.0,0.7,2.1)$ shown in Fig. 12-(b) is slightly higher than the measured data, and hence the concentration level on the symmetry plane is slightly under-predicted.

The calculated $u$-velocity, concentration, and turbulent kinetic energy contours are shown in Figs. 12-(a), (b), and (c), respectively. It can be found in Crabb et al. [1] that the present numerical results are in excellent agreement with the experimentally obtained contour plots. The slight difference between the calculated and the measured u-velocity contour plots in the vicinity of $z / D=4.5$ is again attributed to the coarse grid inaccuracy in the region. Note that the peak concentration occurs in the region where u-velocity is minimum. This trend indicate that the turbulent transport of the concentration is significantly different from that of the momentum. The concentration contour plot exhibits a strong similarity with the turbulent kinetic energy contour plot. This trend is also in excellent agreement with the experimentally observed distributions of the concentration and the turbulent kinetic energy [1]. The excellent agreement between the calculated and the measured contour plots indicates that the M-S turbulence model can correctly resolve the turbulent transports of mass and momentum and that the turbulent transport of mass and momentum depends strongly on the inequilibrium turbulence. It is not clear as yet if any single-time-scale turbulence model can correctly resolve the concentration field as the M-S turbulence model can.

The three-dimensional particle trajectories are shown in Fig. 13. It can 
be seen in the figure that the fluid particles passing near the jet exit are most easily entrained to the jet. It is also shown in the figure that the fluid particles near the jet edge carry less momentum and hence these particles are quickly entrained to the helical vortices in the wake region of the jet. The particle trajectories show that the large eddy mixing occurs in the wide region of the jet edge and that the fluid particles in the center region of the jet does not mix easily with the crossflow. The concentration profiles shown in Fig. 12 also indicate the same trend of the large eddy mixing.

\section{CONCLUSIONS AND DISCUSSION}

It has been shown that a strong inequilibrium turbulence field is characterized by the shape and the frequency domain of the spectral density that varies widely in space. The influence of the inequilibrium turbulence on the development of the mean fluid flow (and consequently, on the development of the turbulence field itself) can be sensed only through semi-empirical data. Thus the influence of the inequilibrium turbulence on the mean fluid flow is more difficult to recognize than other turbulence phenomena such as the anisotropy of turbulence. The semi-empirical data show that the eddy viscosity coefficient becomes smaller in the production dominated region, and becomes larger in the dissipation dominated region. In the multiple-time-scale turbulence model, the dependence of the eddy viscosity coefficient on the inequilibrium turbulence is reflected in the ratio of the dissipation rate $\left(\epsilon_{t}\right)$ to the energy transfer rate $\left(\epsilon_{p}\right)$. In the simplified split-spectrum case, the measured spectral density curves show that the ratio of $k_{p} / k_{t}$ is greater for larger eddies, and becomes smaller as the large eddies cascade down to smaller eddies. The calculated $k_{p} / k_{t}$ 
using the multiple-time-scale turbulence model also shows the same behavior as that observed in experiments. The accurate numerical results for a wide class of complex turbulent flows (including the present jet in the crossflow) obtained using the present multiple-time-scale turbulence model indicate that the turbulent transport of mass, concentration, and momentum depends strongly on the inequilibrium turbulence and that the multiple-time-scale turbulence model correctly resolves the Inequilibrium turbulence phenomena. It is not clear if single-time-scale turbulence models can resolve such inequilibrium turbulence phenomena as yet.

Numerical results for the three-dimensional turbulent flow of a circular jet in a crossflow show that the jet and the crossflow interact very strongly with each other in the forward region of the jet and that the interaction creates a strong inequilibrium turbulence field in the forward region of the jet. The strong interaction between the jet and the crossflow at the jet exit also influences the fluid flow and the turbulence field in the upstream region of the jet. This results suggests that the upstream region of the circular jet needs to be included into the computational domain in order to obtain accurate numerical results or to assess the predictive capability of a turbulence model.

The calculated velocity, concentration, and turbulence fields are in good agreement with the measured data. Both the calculated results and the measured data show that the jet in crossflow is characterized by highly complex velocity, concentration, and turbulence fields that are not usually found in many other turbulent flows. It is discussed in Crabb et al. [1] that the weak vortex shedding does not influence the mean fluid flow significantly. The good comparison between the numerical results and the measured data is also in agreement with such an observation. The calculated 
tangential velocity, concentration, and turbulent kinetic energy contours at a downstream location show that the peak concentration occurs where the tangential velocity becomes local minimum and that the concentration field exhibits a close resemblance to the turbulence fleld. These contour plots are in excellent agreement with the measured contour plots. 


\section{REFERENCES}

1. D. Crabb, D. F. G. Durao and J. H. Whitelaw, "A Round Jet Normal to a Crossflow," Journal of Fluid Engineering, vol. 103, March, 1981, pp. $142-152$.

2. Z. A. Khan, "Opposed Jets in Crossflow," Ph.D. thesis, Mechanical Engineering Dept., Imperial College of Science and Technology, London, 1982.

3. R. W. Claus and S. P. Vanka, "Multigrid Calculations of a jet in Cross Flow," AIAA Paper 90-0444, Aerospace Sclences Meeting, Reno, Nevada, January, 1990.

4. S. V. Patankar, D. Basu and S. Alpay, "Prediction of Three-Dimensional Velocity Field of a Deflected Turbulent jet, " Journal of Fluid Engineering, vol. 99, no. 4, 1977, pp. 758-762.

5. A. J. White, "The Prediction of the Flow and Heat Transfer in the Vicinity of a Jet in Crossflow," ASME Paper A81-21108, 1980.

6. S. Syed and L. M. Chiappeta, "Finite-Difference Methods for Reducing Numerical diffusion in TEACH-Type Calculations," AIAA paper 85-0057, 1985.

7. J. Andreopoulos, "Measurements in a Jet-Pipe Flow Issuing Perpendicularly into a Cross Stream," Journal of Fluid Engineering, vol. 104, December, 1982, pp. 493-499.

8. S. J. Kline, B. J. Cantwell and G. M. Lilley, The 1980-1981 AFOSR-HTTM Stanford Conference on Complex Turbulent Flows, vols. 1-3, Stanford University, Stanford, California, 1981.

9. Kim, S.-W. and Chen, C.-P., "A Multiple-Time-Scale Turbulence model Based on Variable Partitioning of the Turbulent Kinetic Energy Spectrum", Numerical Heat Transfer, Part B, Vol. 16, 1989, pp. 193-211. 
10. S.-W. Kim, "Numerical Investigation of an Internal Layer in Turbulent Flow over a Curved Hill," To appear in Numerical Heat Transfer, also available as NASA TM-102230, 1989.

11. S.-W. Kim, "Numerical Investigation of Separated Transonic Turbulent Flows with a Multiple-Time-Scale Turbulence Model," Numerical Heat Transfer, Part A, vol. 18, 1990, pp. 149-171.

12. S. W. Kim, "Calculation of Reattaching Shear Layers in Divergent Channel with a Multiple-Time-Scale Turbulence Mode1," To appear in AIAA Journal, also available as AIAA Paper 90-0047, 1990.

13. S.-W. Kim, "A Near-Wall Turbulence Model and Its Application to Ful:Developed Turbulent Channel and Pipe Flows," Numerical Heat Transfer, Part B, Vol. 17, 1990., pp. 101-122.

14. S. V. Patankar, Numerical Heat Transfer and Fluid Flow, McGraw-Hill, New York, 1980.

15. S. -W. Kim, "Calculations of Separated 3-D Flows with a Pressure-Staggered Navier-Stokes Equations Solver," NASA CR, In print, 1990.

16. S.-W. Kim, "On the Anomaly of Velocity-Pressure Decoupling in Collocated Mesh," NASA TM, In print, 1990.

17. M. Peric, R. Kessler and G. Scheurerer, "Comparison of Finite-Volume Numerical Methods with Staggered and Collocated Grids," Computers a: id Fluids, vol. 16, no. 4, pp. 389-403, 1988.

18. S. Majumdar, "Role of Underrelaxation in Momentum Interpolation fnr Calculation of Flow with Nonstaggered Grids," Numerical Heat Transfer. vol. 13, Pp. $125-132,1988$.

19. P. S. Klebanoff, "Characteristics of Turbulence in a Boundary Layer with Zero Pressure Gradient," NACA Report 1247, 1955. 
20. I. Wygnanski and H. Fiedler, "Some Measurements in the Self-Preserving Jet," Journal of Fluid Mechanics, vol. 3, part 3, 1969, pp. 577-612.

21. W. Rodi, The Prediction of Free Boundary Layers by Use of a Two-Equation Model of Turbulence," Ph.D. Thesis, University of London, London, 1972.

22. B. E. Launder, "A Generalized Algebraic Stress Transport Hypothesis," AIAA Journal, vol. 20, 1982, pp. 436-437.

23. S.-W. Kim and Y.-S. Chen, "A Finite Element Calculation of Turbulent Boundary Layer Flows with an Algebraic Stress Turbulence Model," Computer Methods in Applied Mechanics and Engineering, vol. 66, no. 1, January, 1988, pp. 45-63.

24. K. Hanjelic, B. E. Launder and R. Schiestel, "Multiple-Time-Scale Concepts in Turbulent Shear Flows" in L. J. S. Bradbury, F. Durst, B. E. Launder, F. W. Schmidt and J. H. Whitelaw, (eds.), Turbulent Shear Flows, Vol. 2, Springer-Verlag, New York, 1980, pp. 36-49.

25. V. G. Harris, J. A. H. Graham and S. Corrsin, "Further Experiments in Nearly Homogeneous Turbulent Shear Flow," Journal of Fluid Mechanics, Vol. 81, 1977, pp. 657-687.

26. S. Tavoularis and U. Karnik, "Further Experiments on the Evolution of Turbulent Stresses and Scales in Uniformly Sheared Turbulence, " Journal of Fluid Mechanics, vol. 204, 1989, pp. 457-478. 


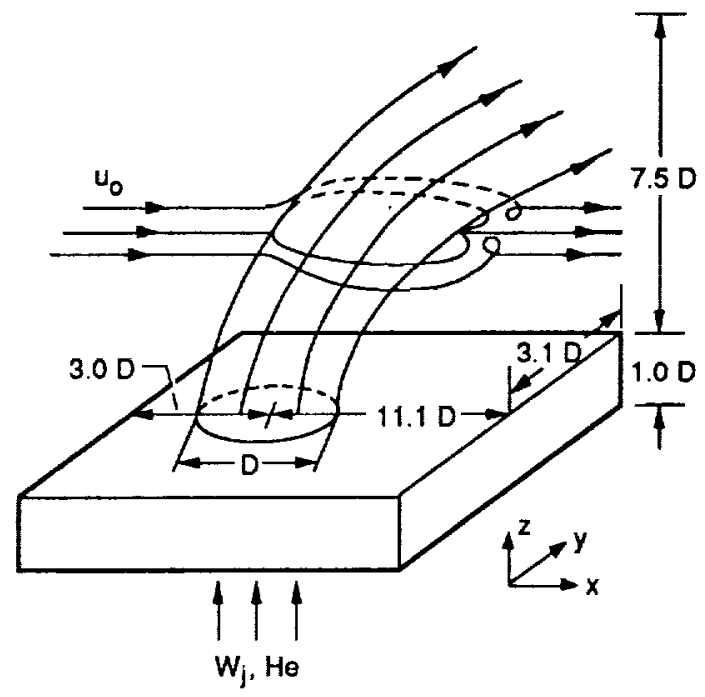

Figure 1.-Nomenclature and computational domain for a circular jet in crossflow.

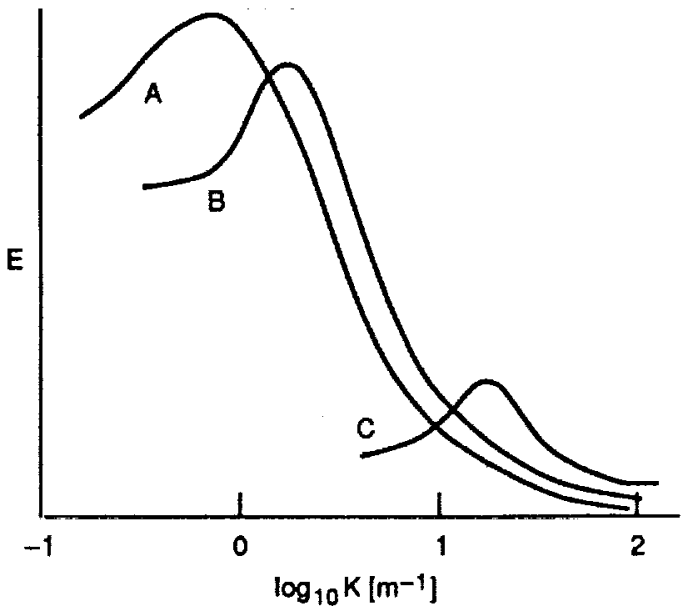

(a) Spectral density for inequilibrium turbulent flows. A: maximum shear location in a circular jet [20],

B: center of a circular jet [20], C: free stream region

of a boundary layer flow in zero pressure gradient [19]

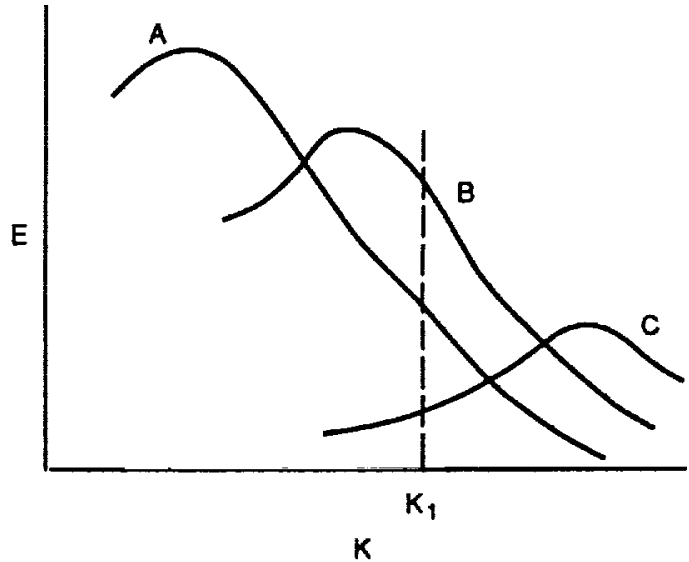

$$
k_{p}=\int_{K=0}^{K_{1}} E d K, \quad k_{t}=\int_{K=K_{1}}^{\infty} E d K
$$

(b) $k_{p} / k_{t}$ for inequilibrium turbulent flow, A: $P r / \epsilon_{t}>1$, $B: P r / \epsilon_{t}=1, C: P r \approx 0.0$.

Figure 2.-Spectral density. 


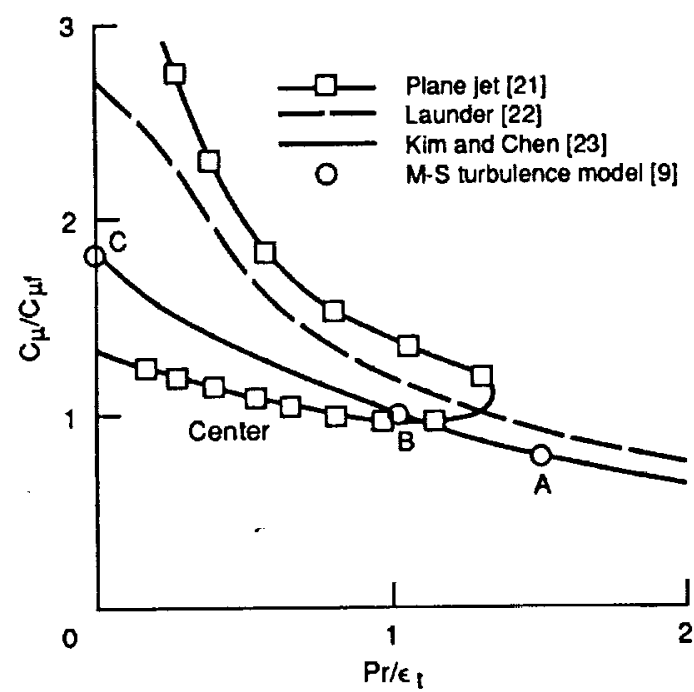

Figure 3. $-c_{\mu} / c_{\mu f}\left(=\epsilon_{l} / \epsilon_{p}\right)$ profiles, $A: P r / \epsilon_{t}>1$. $B: P r / \epsilon_{t}=1, C: P r=0.0$

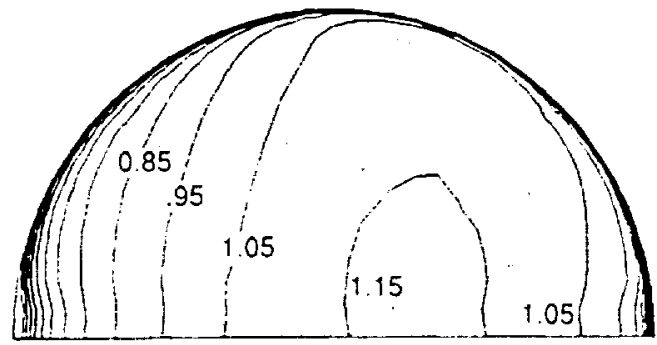

(a) Jet velocity, $w W_{j}$.

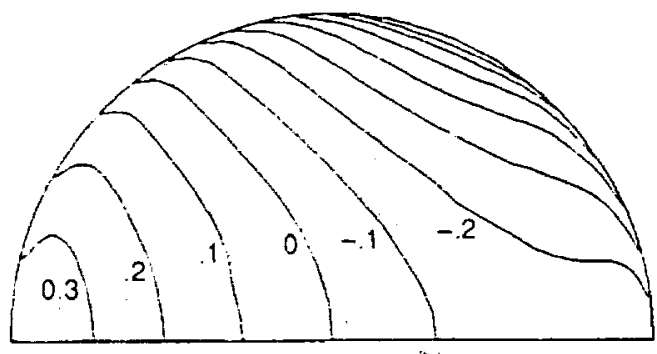

(b) Pressure, $\mathrm{p} / 0.5 \mathrm{pW} \mathrm{j}_{\mathrm{j}}^{2}$.

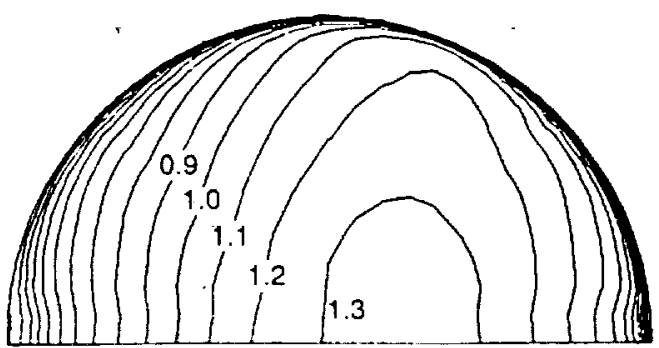

(c) Total pressure $\left(p+0.5 \rho w^{2}\right) / 0.5 \rho W_{j}^{2}$.

Figure 5.-Contour plots of the flow field at the jet exit.

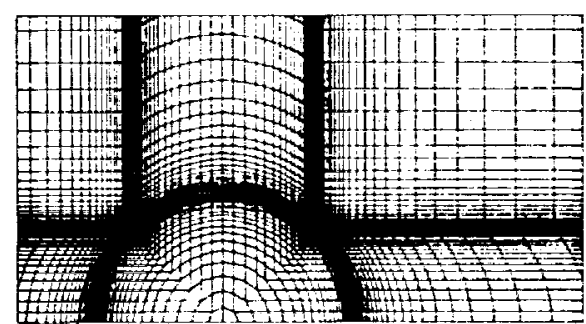

(a) Top View.

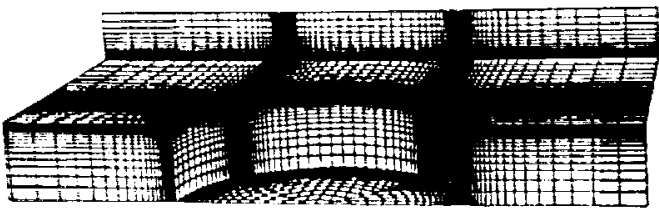

(b) Perspective view.

Figure 4.-Mesh in the vicinity of jet exit.

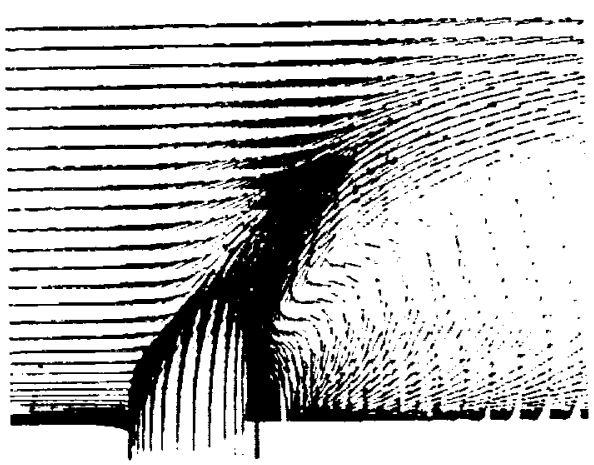

(a) Velocity vector.

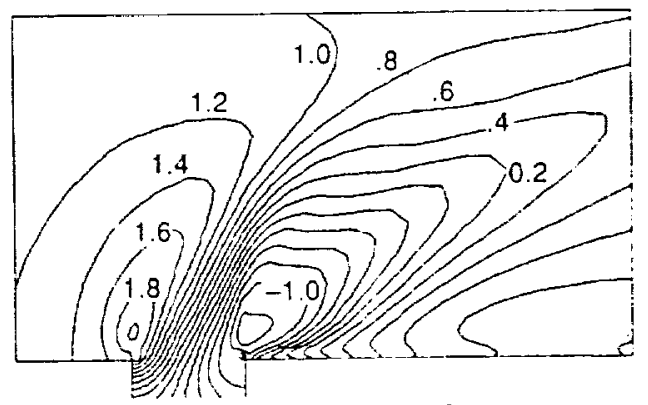

(b) Pressure, $p / 0.5 p \mathrm{U}_{\infty}^{2}$

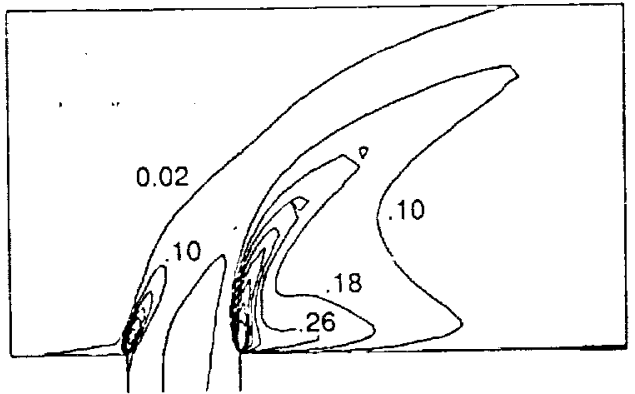

(c) Turbulent kinetic energy $\left(\mathrm{k} / 0.5 \mathrm{U}_{0}^{2}\right)$.

Figure 6.-Flow and turbulence fields on the symmetry plane. 


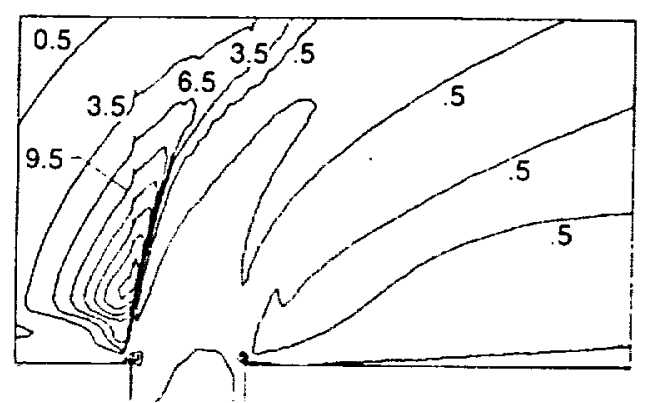

(d) Pr/er.

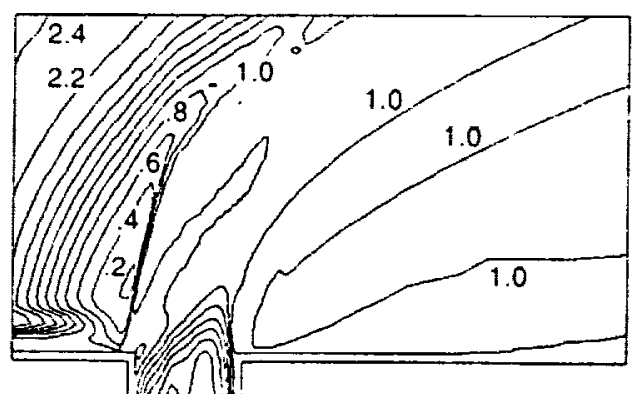

(e) $\epsilon_{\mathrm{t}} / \epsilon_{\mathrm{p}}$.

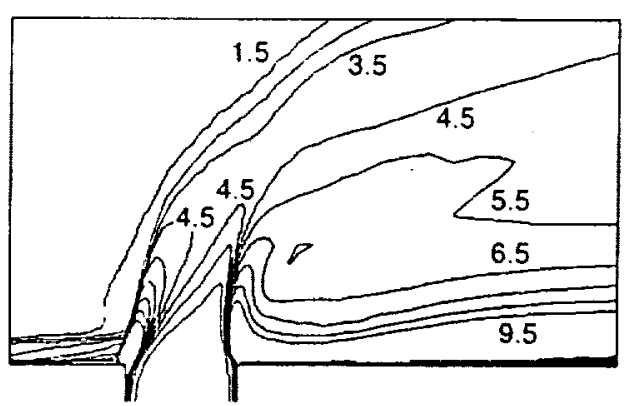

(i) $k_{\mathrm{p}} / \mathrm{k}_{\mathrm{t}}$.

Figure 6.-Concluded.

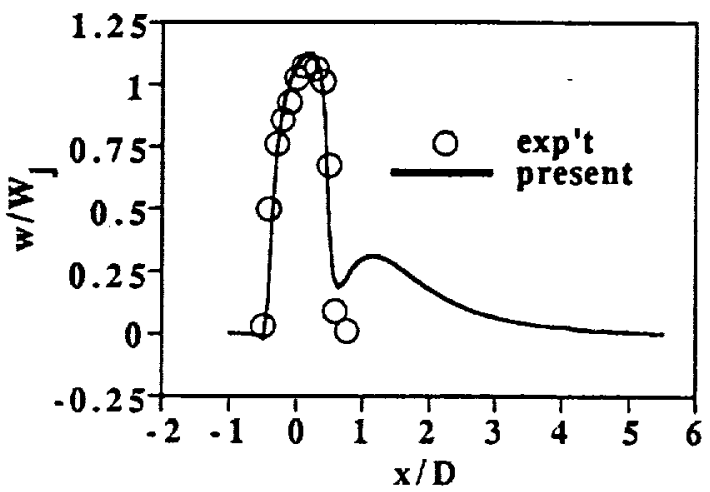

(a) $z / D=0.25$.

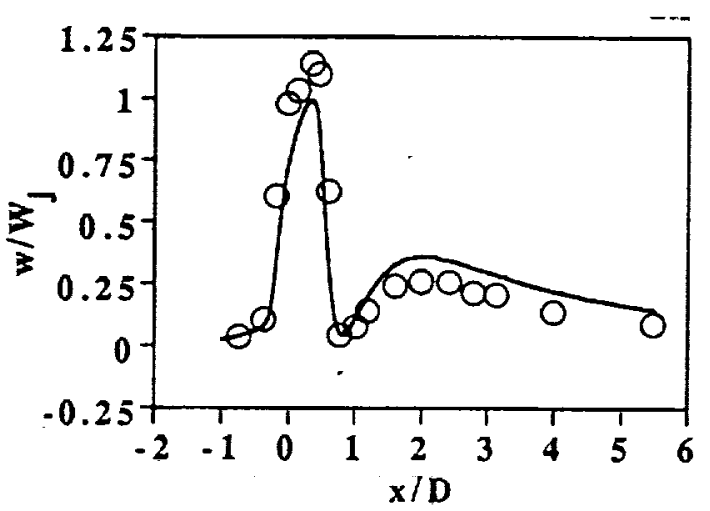

(b) $2 / D=0.75$.

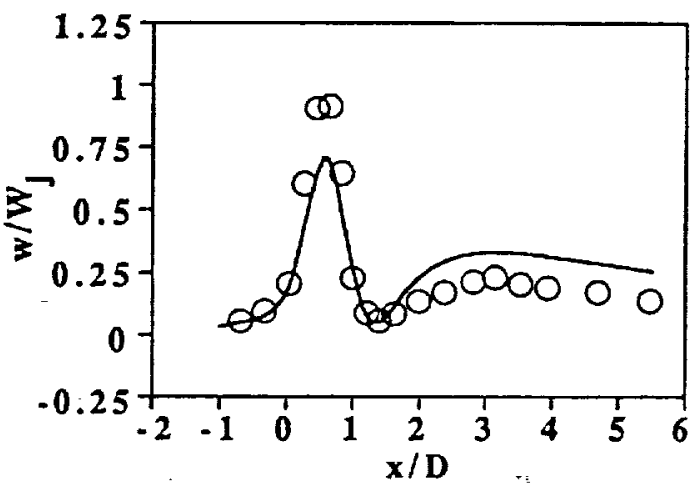

(c) $z / D=1.35$.

Figure 7.-Normal velocity (w) profiles on the symmetry plane. 


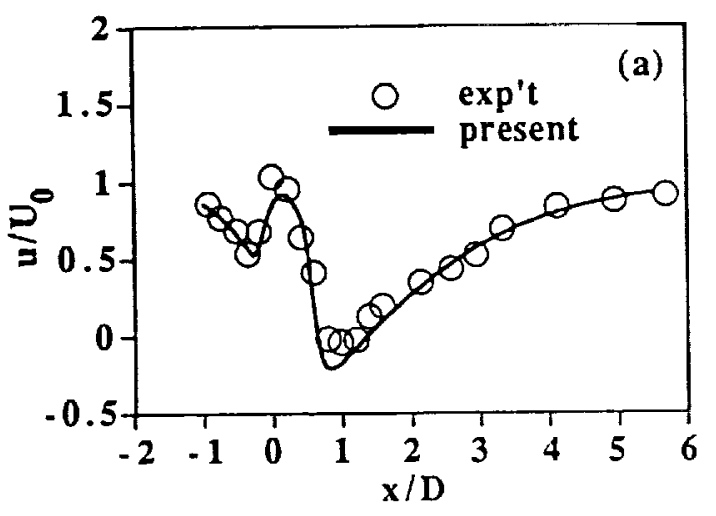

(a) $\mathrm{z} / \mathrm{D}=0.75$.

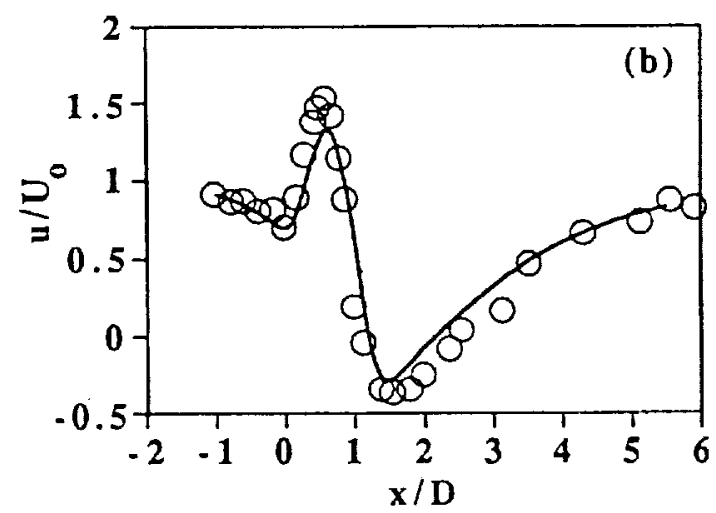

(b) $z / D=1.35$.

Figure 8.-Tangential velocity $(u)$ profiles on the symmetry plane.

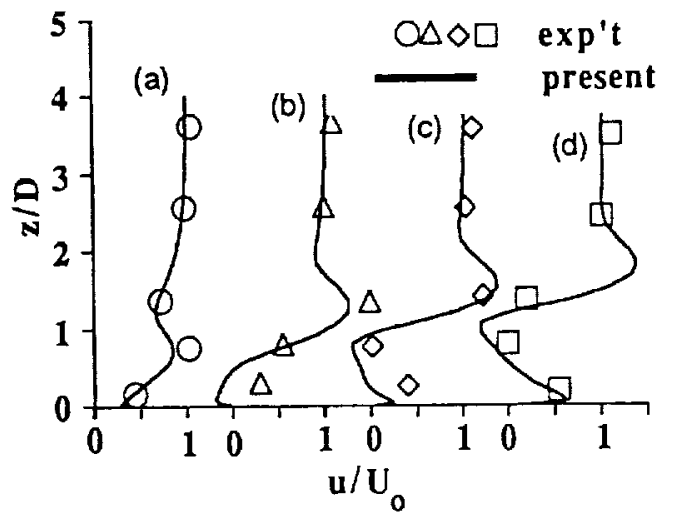

Figure 9.-Tangential velocity (u) profiles on the symmetry plane, (a) $x / D=0$,

(b) $x / D=0.5$, (c) $x / D=0.75$, (d) $x / D=$ 1.0 .

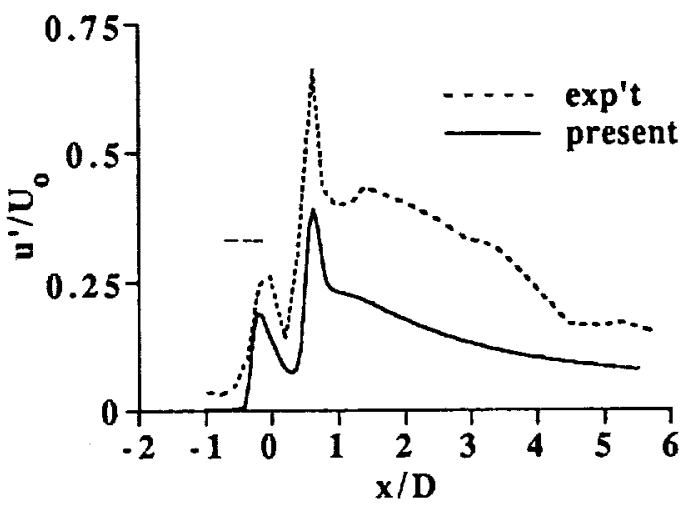

Figure 10.-Turbulent kinetlc energy profile on the symmetry plane at $z / D=0.75$.

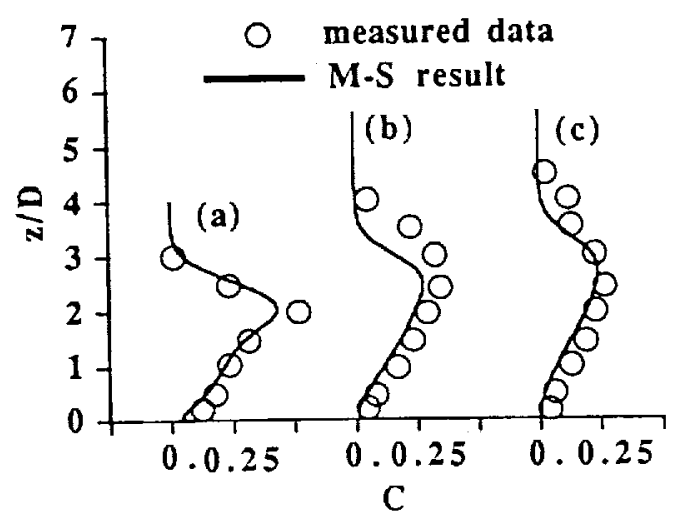

Figure 11.-Concentration profiles at downstream locations on the symmetry plane, (a) $x / D=4$, (b) $x / D=6$, (c) $x / D=$ 8. 

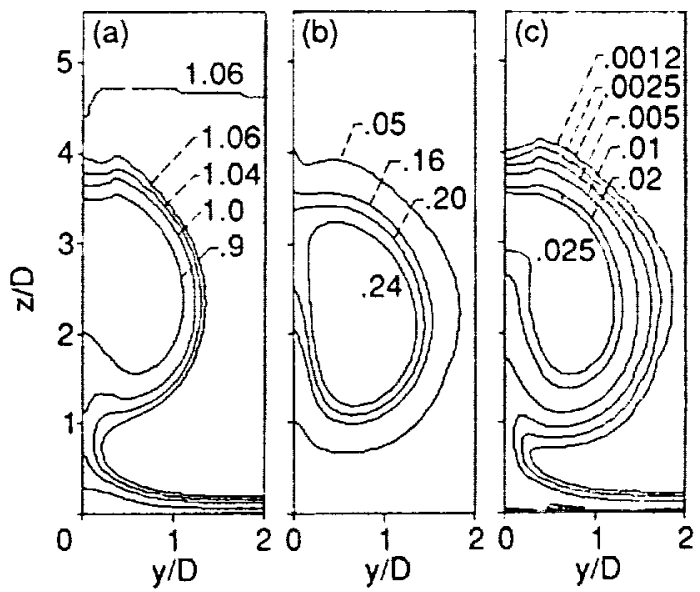
(a) u-velocity.
(b) Concentration.
(c) Turbulent
kinetic
energy.

Figure 12.-Contour plots of u-velocity, concentration and turbulent kinetic energy.

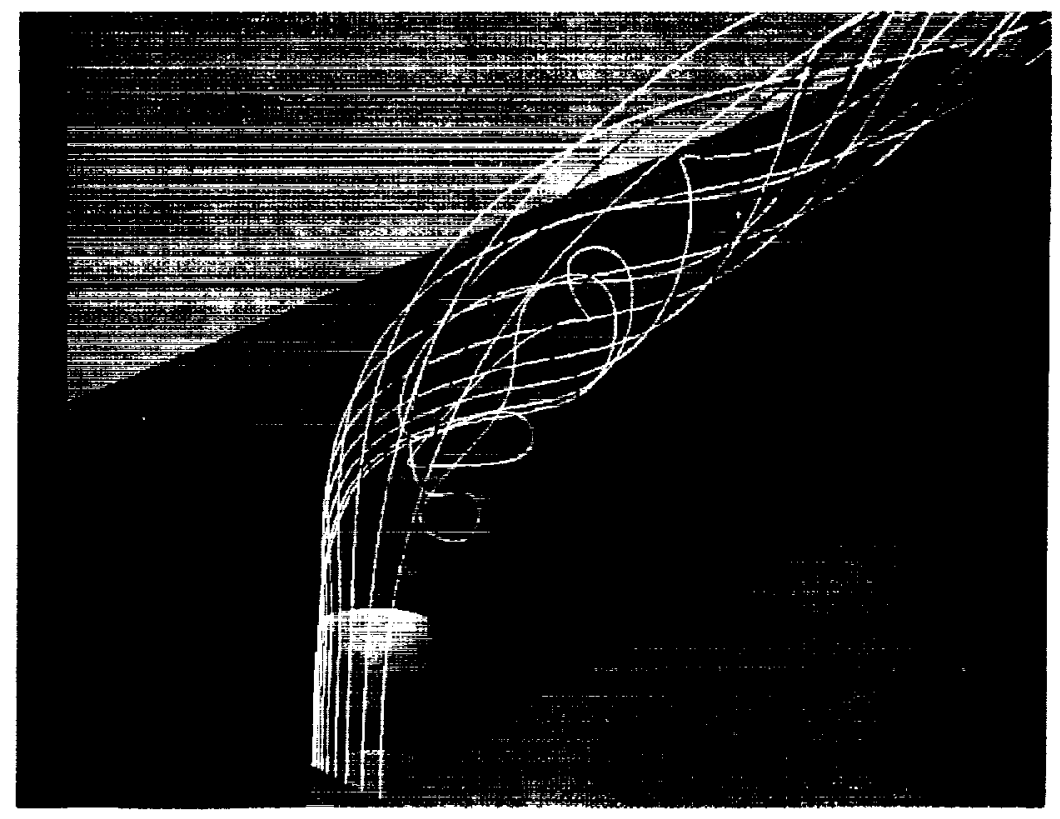

Figure 13.-Three-dimensional particle trajectories of a jet in a crossflow. 


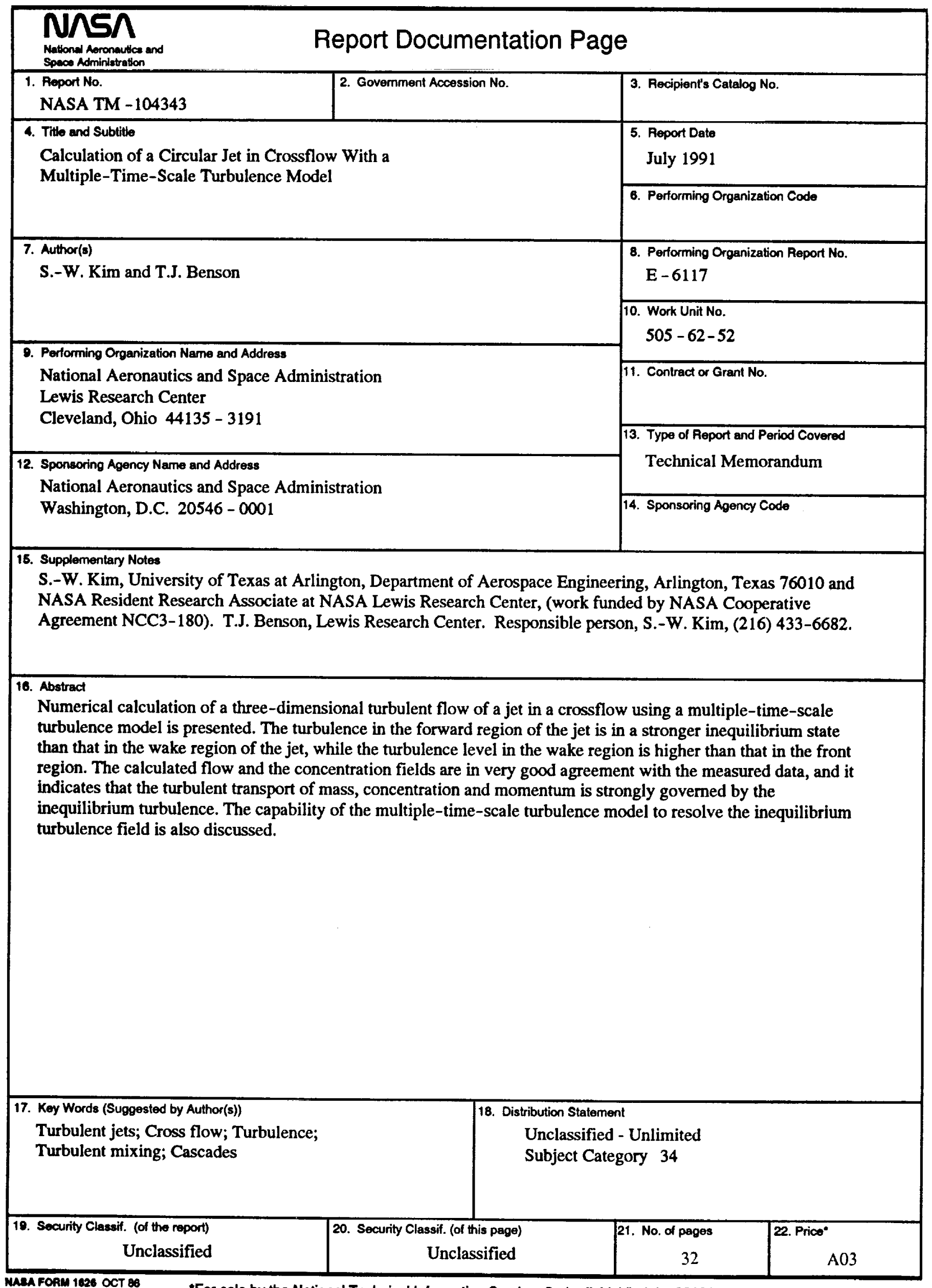

Perlorming Organizat

Lewis Research Center

Cleveland, Ohio 44135 - 3191

Technical Memorandum

National Aeronautics and Space Administration

Washington, D.C. $20546-0001$

14. Sponsoring Agency Code

Supplementary Notes

NASA Resident Research Associate at NASA Lewis Research Center, (work funded by NASA Cooperative

Agreement NCC3-180). T.J. Benson, Lewis Research Center. Responsible person, S.-W. Kim, (216) 433-6682.

\section{Abstract} than that in the wake region of the jet, while the turbulence level in the wake region is higher than that in the front ing . The calculated flow and the concentration fields are in very good agreement with the measured data, and it inequilibrium turbulence. The capability of the multiple-time-scale turbulence model to resolve the inequilibrium urbulence field is also discussed. 
